Title:

A stand-alone compact EUV microscope based on gas-puff target source

Authors:

Alfio Torrisi, Przemyslaw Wachulak, Łukasz Węgrzyński, Tomasz Fok, Andrzej Bartnik, Tomáš Parkman, Šárka Vondrová, Jana Turňová, Bartłomiej J. Jankiewicz, Bartosz Bartosewicz and Henryk Fiedorowicz

\title{
Final manuscript
}

The original publication may be found at:

Journal: Journal of Microscopy 265, 251-260 (2017)

DOI: http://dx.doi.org/10.1111/jmi.12494 


\title{
A stand-alone compact EUV microscope based on gas-puff target source
}

\author{
Alfio Torrisi ${ }^{*}$, , Przemyslaw Wachulak ${ }^{1}$, Łukasz Węgrzyński ${ }^{1}$, Tomasz Fok ${ }^{1}$, Andrzej Bartnik ${ }^{1}$, \\ Tomáš Parkman ${ }^{2}, \breve{S}^{\prime}$ rka Vondrová ${ }^{2}$, Jana Turňová ${ }^{2}$, Bartłomiej J. Jankiewicz ${ }^{1}$, Bartosz Bartosewicz ${ }^{1}$ \\ and Henryk Fiedorowicz ${ }^{1}$ \\ ${ }^{1}$ Institute of Optoelectronics, Military University of Technology, \\ Kaliskiego 2 Str., 00-908 Warsaw, Poland \\ ${ }^{2}$ Czech Technical University in Prague, Faculty of Biomedical Engineering, \\ 27201 Kladno, Czech Republic \\ *corresponding author: alfio.torrisi@ wat.edu.pl \\ phone: +4822 261839609 , fax: +48226668950
}

\begin{abstract}
We report on a very compact desk-top transmission extreme ultraviolet (EUV) microscope based on a laser-plasma source with a double stream gas-puff target, capable of acquiring magnified images of objects with a spatial (half-pitch) resolution of sub-50 $\mathrm{nm}$. A multilayer ellipsoidal condenser is used to focus and spectrally narrow the radiation from the plasma, producing a quasi-monochromatic EUV radiation $(\lambda=13.8 \mathrm{~nm})$ illuminating the object, while a Fresnel zone plate objective forms the image. Design details, development, characterization and optimization of the EUV source and the microscope are described and discussed. Test object and other samples were imaged to demonstrate superior resolution compared to visible light microscopy.
\end{abstract}

\section{KEYWORDS:}

EUV microscopy, Fresnel zone plate, Gas-puff target, EUV source, Imaging.

\section{INTRODUCTION}

Progress in sciences, especially nanotechnology, requires improvement of the microscopy spatial resolution in order to obtain images in the nanometer scale. According to the Rayleigh criterion, light of shorter wavelength can be focused to smaller volume - foci, improving the diffraction limited resolution and making possible to see finer details in high spatial resolution images.

Extreme ultraviolet (EUV, often referred as XUV, wavelength $\lambda=10-120 \mathrm{~nm}$ ) and soft X-Rays (SXR, $\lambda=0.1-10 \mathrm{~nm}$ ) radiations for microscopy investigations allow to obtain additional information about the sample's composition and morphology, not directly available through the use of optical microscopy or scanning electron microscopy (SEM). The EUV radiation can be easily absorbed by solid materials with very small thicknesses of the order of about $100 \mathrm{~nm}$ (Wachulak et al., 2007) and by gaseous materials with thicknesses of the order of a few millimeters. Moreover, such radiation can provide information about such thin materials, depth of the micro-cracks (Wachulak et al., 2012) or directly visualize the gas flow in 2-D (Wachulak et al., 2015a) and 3-D mode (Wachulak et al., 2014), usually investigated with indirect techniques such as interferometry (Park et al., 2013). In order to obtain a sufficiently high number of energetic photons, which is required for the EUV microscopy, it is necessary to employ sources capable of reaching a high photon flux (Hädrich et al., 2015; Ojeda et al., 2016), such as free electron lasers (Yase et al., 2013) and synchrotrons (Hädrich et al., 2014). While they are the brightest sources of soft X-rays and 
the EUV, these facilities are expensive, require high maintenance costs, because of their scale and complexity. Moreover, they provide a limited user access. In order to overcome these limitations, recent researches led to the development of a new generation of compact EUV and SXR sources, which are more adequate for compact, lab-based systems and less expensive than large facilities.

Different compact EUV sources are currently available to perform sub-micrometer and nanometer image acquisitions using different approaches. With the radiation from a capillary discharge laser source, operating at a wavelength of $\lambda=46.9 \mathrm{~nm}$, EUV images can have a spatial resolution from $150 \mathrm{~nm}$ (Vaschenko et al., 2005) down to $\sim 50 \mathrm{~nm}$ (Wachulak et al., 2008), and holographic images down to sub-50 nm (Wachulak et al., 2009). A $13.2 \mathrm{~nm}$ wavelength radiation from Ni-like Cd EUV laser was shown to enable EUV nano-imaging of various samples with a sub-38 nm spatial resolution in transmission mode, and a picosecond time resolution (Vaschenko et al., 2006). However, due to relatively short lifetime of the capillaries, the number of acquired images is also limited (Klosner \& Silfvast, 1998). Nevertheless, such systems are much more compact and can be easily implemented in laboratory environment. Progress in the optimization of gaseous plasma sources to obtain high resolution imaging has also been achieved, employing different techniques, such as a single, nitrogen stream gas-puff target (Müller et al., 2014), high-harmonic generation (Tadesse et al., 2016) and supersonic jets (Mey et al., 2012).

Since Fresnel zone plates (FZPs) optics were proposed for imaging experiments (Baez, 1952a; Baez, 1952b), they have been extensively used in many previously mentioned experiments and with various radiation wavelengths, from the visible (Baez, 1961), EUV (Wachulak et al., 2010a, Zhang et al., 2013, Benk et al., 2015) and soft X-rays (Vaschenko et al., 2006) to hard X-ray radiation (Döring et al., 2013), in various imaging techniques such as bright (Jones et al., 2014) and dark field (Simons et al., 2015), scanning microscopy (Thibault et al., 2008) and, more recently, phytography (Wilke et al., 2012), micro (Mizutani et al., 2013) and nanotomography (Saiga et al., 2016).

During the last few years, many efforts were made to improve the EUV and SXR microscopy using FZPs and compact sources. Although current zone plates are capable of achieving $\sim 10 \mathrm{~nm}$ spatial resolution (Rehbein et al., 2009), the sources are still quite a challenge to overcome due to the requirements of high photon flux and repetition rate, low or none debris production, compact construction, etc. These compact sources represent an important alternative to perform experiments in small academic laboratories and, following to a widespread and feasible commercialization, may have a huge impact, speeding up development of nanotechnology in the near future.

One of the compact, desk-top, short wavelength sources that are capable of being a driver for high-resolution microscope is a laser-plasma source based on a double stream gaspuff target. This source is very robust, simple in construction, has low maintenance costs and enables the performing of experiments with many advantages, namely a high photon flux, obtaining a monochromatic radiation emission, a change in the emission spectrum simply by changing the gas - target, or tuning the emission to one's needs by applying different thin film filters. The gas-puff target source has already been proven to be suitable and, recently, it was successfully used in a variety of imaging experiments including SXR full field microscopy obtaining half-pitch spatial resolution up to $60 \mathrm{~nm}$ (Wachulak et al., 2015b). Additionally, the EUV radiation was already shown to be useful for imaging of non-trivial samples, to demonstrate that it can reveal additional information about the sample compared to other microscopes, such as optical microscope and SEM cannot (Wachulak et al., 2013).

Even though the recent development of short wavelength microscopes has made them capable of obtaining very high spatial resolution images, there is still a trade-off, which can be seen between the exposure time and the size and complexity of the microscope's 
architecture. Samples with resolution 35-40 nm were imaged employing a nitrogen cryo-jet target (Legall et al., 2013). On the other hand, this source is complicated and the set-up may occupy a few optical tables. Other compact systems, employing a single gas stream jet, while simple in construction, have the limitation of acquiring $\sim 100 \mathrm{~nm}$ resolution microscopy images with very high exposure times, up to 1-2 hours (Müller et al., 2014). Therefore, the actual challenge is to deal with exposure time and high resolution of the systems. Moreover, a desk-top size set-up offers the opportunity of commercializing such compact microscopes in the future.

In this paper we present a recently developed compact stand-alone EUV microscope, which is a closed architecture system. The design is based on results from our previous experimental system (Wachulak et al., 2012). The EUV microscope is based on a laserplasma double stream gas-puff target source, which permits to reach a sub-50 nm spatial resolution in a few-second exposure time. To our knowledge, such EUV microscope is one of the most compact microscopes reported in the literature. The source employed shows many advantages compared with other sources used for similar microscopy applications and opens the way to future commercialization. Most importantly, the employment of a gaseous source eliminates the debris production associated with solid targets, since no ablation products are produced during the plasma formation. Moreover, the source is very compact, its construction is very simple, and the maintenance and service costs are low.

\section{EXPERIMENTAL SETUP}

A scheme of the double stream gas-puff target EUV source is shown in Fig. 1a). An infrared Nd:YAG laser pulse (NL 302 from Eksma, Lithuania) operating at $\lambda=1064 \mathrm{~nm}$, with $500 \mathrm{~mJ}$ energy, $4 \mathrm{~ns}$ time duration of the pulses and intensity of $\sim 10^{11} \mathrm{~W} / \mathrm{cm}^{2}$ in a laser focus (focal spot radius $0.1 \mathrm{~mm}$ ), was focused using a lens ( $\mathrm{f}=25 \mathrm{~mm}$ ) onto a double-stream gas-puff target, produced by an electromagnetic double nozzle valve system.

The gaseous target is composed of two circularly symmetric nozzles. The inner nozzle, $0.4 \mathrm{~mm}$ in diameter, injects a small amount of working gas (argon) into the vacuum. The outer nozzle (0.7-1.5 mm in diameter) injects helium, in order to reduce the working gas density gradient along the nozzle axis. In order to form the plasma without damaging the nozzles and sacrificing the efficiency of the EUV photon production, the focal spot is located $1.5 \mathrm{~mm}$ away from the nozzle plane. Additionally, proper time synchronization is required between the gas-puff target formation and the arrival of the laser pulse. When those conditions are satisfied, the valve and the nozzles can operate in synchronization, continuously for millions of laser pulses without any damage to the nozzle. Furthermore, the EUV emission can be used continuously, providing high flux, due to the possibility to operate with high repetition rate laser. To do so, the proper time delay between the synchronization pulse from the pumping laser power supply and the opening times of the two valves supplying two nozzles (with Ar and $\mathrm{He}$ ) are adjusted to be $100 \mu \mathrm{s}$ and $230 \mu \mathrm{s}$, respectively. The duration of time for which the valves stay open - pulse width, are adjusted to be $500 \mu \mathrm{s}$ for both valves. Moreover, the time between the synchronization pulse and the laser pulse, generating the plasma, is set to $1 \mathrm{~ms}$. The gas-puff target approach has been developed for EUV metrology applications in the frame of MEDEA + project (Fiedorowicz et al., 2000; Fiedorowicz et al., 2005), at the Institute of Optoelectronics, Military University of Technology, Poland. More details about the EUV source based on the gas-puff target and its optimization for microscopy applications are reported in the literature (Bartnik et al., 2014; Wachulak et al., 2015c).

The optimum Ar/He pressure was found to be 10 bar and 6 bar, respectively. Each valve was driven independently using a dedicated controller. In order to avoid high gas pressure 
build-up inside the vacuum chamber, a differential pumping was employed. A cone-shaped differential pumping aperture $(\sim 1 \mathrm{~mm}$ in diameter) was used to separate the pressure inside the source chamber (equal to a few mbar during the source operation) from the pressure inside the microscope chamber. In this chamber, shown in Fig. 1b,c), the condenser and other EUV microscope components are located and a pressure down to $10^{-5} \mathrm{mbar}$ can be achieved. Two roughing (scroll) pumps and one turbomolecular pump were used for the pumping, in order to maintain a constant pressure of $\sim 10^{-4}$ mbar during the tests.

In order to collect the radiation emitted from the Ar plasma and to spectrally narrow down the emission, the EUV microscope was equipped with an ellipsoidal off-axis condenser mirror (MLM), located at the distance of $254 \mathrm{~mm}$ from the plasma. The condenser reflects radiation at the wavelength of $13.5 \pm 0.5 \mathrm{~nm}$ (full width half maximum, FWHM) and it is corrected for the spherical aberrations for the incidence angle of $45^{\circ}$. An $80 \mathrm{~mm}$ in diameter ellipsoidal silica mirror (Reflex s.r.o. company, Czech Republic), with radii of $179.6 \mathrm{~mm}$ and $254 \mathrm{~mm}$ in two orthogonal directions, was used as substrate for the deposition of the Mo/Si multilayers, which was performed at the Fraunhofer Institut für Angewandte Optik und Feinmechanik (IOF) in Jena, Germany. The condenser produces an image of the plasma with a magnification of $\mathrm{M}=1$. By using such piece of optic, a quasi-monochromatic EUV radiation was produced and focused at the second focal plane of the condenser, where the object was placed. Moreover, off-axis geometry significantly reduces the overall footprint of the entire system, which can be seen in Fig. 1c,d).

To eliminate longer wavelengths $(\lambda>16 \mathrm{~nm})$, a $250 \mathrm{~nm}$ thick, $10 \mathrm{~mm}$ in diameter, freestanding $\mathrm{Zr}$ filter (Lebow) was used. The $\mathrm{Zr}$ filter was positioned $\sim 35 \mathrm{~mm}$ upstream the object. The object was located in the second focal plane of the ellipsoidal condenser mirror, at the distance of $254 \mathrm{~mm}$ from the condenser. Three axes remotely driven step motor stages, (8MT173-20) from Standa, Lithuania, were used to adjust the position of the object while the imaging was performed. The positioning accuracy of those stages was $1.25 \mu \mathrm{m}$, according to manufacturer's specifications. The object, locked in place by means of a magnetic (kinematic) holder, manufactured by Thorlabs (KB25/M), was imaged in transmission mode, using a Fresnel zone plate (diameter $D_{F Z P}=200 \mu \mathrm{m}$, number of zones $N_{F Z P}=1000$ and outer zone width $\Delta r=50 \mathrm{~nm}$ ) onto a EUV sensitive CCD camera, iKon-M (Andor), with $1024 \times 1024$ pixels and $13 \times 13 \mu \mathrm{m}^{2}$ pixel size. The FZP was fabricated by Zone Plates Ltd., using electron beam lithography in a $200 \mathrm{~nm}$ thick PMMA (polymethyl methacrylate) layer spin coated on top of a $50 \mathrm{~nm}$ thick $\mathrm{Si}_{3} \mathrm{~N}_{4}$ membrane. Such zone plate, has relatively large diffraction efficiency, comparing to other FZPs made in different materials, reaching $20 \%$ in the first diffraction order. A comparison plot for various possible materials often used for zone plate fabrication is shown in Fig. 2. The calculation is based on (Kirz, 1974). For a quasi-monochromatic radiation at $\lambda=13.84 \mathrm{~nm}$ from Ar plasma, the focal length of the FZP was equal to $\mathrm{f}=722.5 \mu \mathrm{m}$, providing a theoretical spatial resolution (Rayleigh resolution or full-period resolution) of $61 \mathrm{~nm}$ and a depth of focus (DOF) equal to $\pm 369 \mathrm{~nm}(738 \mathrm{~nm}$ in total). Moreover, the numerical aperture of the condenser $\left(\mathrm{NA}_{\mathrm{CH}}=0.11\right.$ in horizontal and $\mathrm{NA}_{\mathrm{CV}}=0.15$ in vertical direction) matches with the numerical aperture of the FZP objective $\left(\mathrm{NA}_{\mathrm{o}}=0.137\right)$, thus providing spatially incoherent object illumination (Heck et al., 1998). The magnification can easily be increased by adjusting the FZP to CCD distance. A second set of motorized stages was used to position the FZP objective. Additionally, for more accurate positioning of the FZP along the optical axis of the EUV microscope, within a fraction of the DOF of the FZP, a dedicated piezo stage (LPS-45) from PI miCos GmbH, Germany, was used, thus having a repeatability of $50 \mathrm{~nm}$. Additionally, the chamber is provided with a supplementary load-lock in order to easily change the sample.

The mechanical stability of the EUV microscope was improved by separating the vacuum pumps from the microscope and by developing a dedicated stable supporting rack for the 
entire system. The bottom plate was isolated from the bottom of the chamber in order to avoid any possible small shifts and thermal drifts of the internal components that could influence the refocusing accuracy limited by the depth of focus of the FZP.

Moreover, the user's safety was improved. Indeed, due to the fact that the Nd:YAG laser is directly attached to the source, the laser beam does not propagate outside the microscope chamber, therefore the user doesn't need to use protective glasses. Fig. 1c) shows the internal components of the microscope, located in the vacuum chamber. The entire microscope has a very compact size, $(\mathrm{WxDxH}) 100 \mathrm{~cm} \times 70 \mathrm{~cm} \times 160 \mathrm{~cm}$, which can be seen in the photograph shown in Fig. 1d).

\section{EXPERIMENTAL RESULTS}

\subsection{EUV source measurements}

A $10 \times 10 \mathrm{~mm}^{2}$ AXUV 100 detector from IRD Inc., USA, coated with a thin layer of $\mathrm{Zr}$ $(200 \mathrm{~nm})$ and $\mathrm{C}(50 \mathrm{~nm})$ was employed for photon flux measurements. The detector with such coatings is sensitive to plasma radiation in the wavelength range of 6-16 $\mathrm{nm}$. The detector signal was stored using a $4 \mathrm{GHz}$ Tektronix DPO 70404 oscilloscope and later integrated numerically. The photon flux, estimated in a second focal plane of the condenser, where the object is located, in a 13-14 nm spectral range for a single EUV pulse, was $(1.36 \pm 0.07) \cdot 10^{11}$ photons. The associated flux error was $\sim 5.1 \%$, based on 23 measurements. This results in a photon flux density of $\sim 1.47 \cdot 10^{6}$ photons $/ \mu \mathrm{m}^{2} / \mathrm{sec}$ at a $10 \mathrm{~Hz}$ source repetition rate. The energy of a single pulse in the full solid angle in the spectral range of 13$14 \mathrm{~nm}$ was $1.9 \mathrm{~mJ}$. The pulse energy of the Nd:YAG laser was $500 \mathrm{~mJ}$, so the radiation conversion efficiency from the IR to the $\Delta \lambda=1 \mathrm{~nm}$ EUV narrow band was equal to $0.38 \%$.

The plasma size in the EUV range was measured using a pinhole camera, equipped additionally with a $250 \mathrm{~nm}$ thick $\mathrm{Zr}$ filter, located at a distance of $100 \mathrm{~mm}$ from the pinhole, $32 \mu \mathrm{m}$ in diameter. The geometrical magnification of the pinhole camera was $1.42 \mathrm{x}$. Since the $\mathrm{Zr}$ filter reduces the quantity of photons reaching the detector during acquisition, an exposure of 20 EUV pulses was required to acquire a single image. The measured EUV plasma spatial distribution was elongated in the direction of the incoming Nd:YAG laser beam and its size was estimated to be $0.75 \times 0.38 \mathrm{~mm}^{2}$ (FWHM), as shown in Fig. 3a,c). The brightness of the plasma image, shown in Fig. 3a), was deliberately increased to show the relative position of the plasma and a differential pumping cone aperture. It is shown as a thin symmetrical ring with a diameter of $1.2 \mathrm{~mm}$, created by the scattering of plasma radiation from the pinhole edge. The plasma profile measured in two orthogonal directions is shown in Fig. 3b) and d). Fig. 3c), in "false colors", shows in detail the hottest and the most intense region of the plasma (red color).

Spectral measurements of the laser-plasma source were performed using a $\mathrm{Si}_{3} \mathrm{~N}_{4}$ transmission grating spectrometer with a grating period of $200 \mathrm{~nm}(5000$ lines $/ \mathrm{mm})$. The spectrum was acquired using a CCD camera (X-vision, Reflex, Czech Republic) with an exposure of 20 EUV pulses. A typical spectrum from Ar plasma, obtained directly from the source in a wavelength range of 6-16 $\mathrm{nm}$, employing a $250 \mathrm{~nm}$ thick $\mathrm{Zr}$ filter, is presented in Fig. 4 (straight grey line). The figure shows transmission curve for the multilayer mirror condenser (dashed red line). It also shows the total contribution in the spectral range 13-14 $\mathrm{nm}$ (dash-dotted blue line) of the EUV radiation focused and reflected from the MLM. Only this radiation in a narrow EUV band is used for subsequent imaging. The reflectivity of the MLM condenser was numerically simulated using the Center for X-Ray Optics database (CXRO, http://www.cxro.lbl.gov/). In the $\Delta \lambda=1 \mathrm{~nm}$ wide MLM transmission band the predominant spectral line is at $\lambda=13.84 \mathrm{~nm}$. There are actually two dominant, closely spaced 
spectral lines for $\mathrm{Ar}^{\mathrm{VIII}}$ emission, $2 \mathrm{p}^{6} 3 \mathrm{p}-2 \mathrm{p}^{6} 5 \mathrm{~d}$ at $\lambda=13.793 \mathrm{~nm}$ and more intense $2 \mathrm{p}^{6} 3 \mathrm{~s}$ $2 p^{6} 5 p$ at $\lambda=13.844 \mathrm{~nm}$, (Kelly, 1987). These lines, however, cannot be resolved because the spectrometer spectral resolution was not sufficient to separate such closely spaced lines, only $0.51 \AA$ apart. The FWHM inverse relative bandwidth of the spectrometer used in this study was $\lambda / \Delta \lambda=60$ at $\lambda=13.84 \mathrm{~nm}$, so $\Delta \lambda \sim 2.3 \AA$.

\subsection{Optimization of the EUV condenser position and focal spot imaging}

The condenser position was optimized to obtain the EUV radiation focused in the second focal plane of the condenser. This was done to ensure the maximum energy and a uniform spatial distribution of the illumination on the surface of the sample. To suppress the axial EUV radiation, a circular beam-stop with a diameter of $3.2 \mathrm{~mm}$ was placed $20 \mathrm{~cm}$ downstream the condenser. A $4 \mu \mathrm{m}$ thick P43 scintillator screen, from Proxitronic, deposited on thin glass disk and coated with a $50 \mathrm{~nm}$ Al layer, was placed on a motorized translation stage. It was shifted with $1 \mathrm{~mm}$ steps along the optical axis to obtain a spatial distribution of the EUV radiation emerging from the condenser at different planes. The spatial distributions around the second focal plane of the condenser were subsequently acquired using Canon EOS 350D digital camera, equipped with a telephoto lens. Each image was acquired with $10 \mathrm{EUV}$ pulses and integrated in $\sim 2$ seconds. A few examples of the intensity distributions near the focal plane of the EUV mirror are shown in Fig. 5. The images were acquired at different scintillator positions; the location $\mathrm{z}=0 \mathrm{~mm}$ was assumed to be the focal plane of the MLM condenser. The FWHM size of the spot illuminating the sample was $1.5 \times 0.8 \mathrm{~mm}^{2}$. The plasma variation in size and in intensity at different distances from the condenser focal point can be observed, showing the focusing property of the condenser mirror for the EUV radiation. The smallest achievable spot - central image in Fig. 5, was ellipsoidal, horizontally elongated, in a manner similar to the plasma pinhole camera image (Fig. 3).

\subsection{Image acquisition and knife edge resolution estimation}

As a test object, a copper mesh with a period of $12.5 \mu \mathrm{m}$ and $7.5 \times 7.5 \mu \mathrm{m}^{2}$ square holes was used. The image of the object was acquired by integrating 200 EUV pulses at a source repetition rate of $10 \mathrm{~Hz}$. To increase the photon flux in the sample plane, without sacrificing the spatial resolution, a thinner, $100 \mathrm{~nm}$ thick $\mathrm{Zr}$ filter (Lebow) was used. Its transmission is 1.7 times higher than $250 \mathrm{~nm}$ filter at $13.84 \mathrm{~nm}$ wavelength, according to the data in CXRO database. The magnified image of the object was recorded using Andor DO$934 \mathrm{~N}$ back-illuminated CCD camera, cooled down to $-20{ }^{\circ} \mathrm{C}$ and with a $50 \mathrm{kHz}$ readout rate to minimize the readout noise. A comparison between the images of the test object acquired with the SEM and the EUV microscope is shown in Fig. 6a) and 6b), respectively. The features of the mesh in the EUV image are clearly visible.

To obtain highest spatial resolution EUV image a refocusing procedure was necessary. It was performed by changing the object-objective distance using an LPS-45 piezo closed-loop translation stage with $500 \mathrm{~nm}$ steps along the optical axis. The step size was chosen to be less than the DOF. The "sharpest" EUV image was chosen from series of images in order to determine the spatial resolution of the microscope, assessed with the "knife edge" (KE) test. This test is based on imaging a sharp edge and measuring the distance in the intensity profile for which the intensity varies from 10 to $90 \%$ of the normalized data. For incoherent illumination, such as in this case, this transition distance corresponds to a well-known Rayleigh resolution limit and to twice the value of half-pitch grating resolution of the optical system. The KE test was performed in the region indicated with two green markers in Fig. 6b). The distance between the markers was $2 \mu \mathrm{m}$, which corresponds to $\sim 61$ 
pixels in the EUV image. Consequently, each pixel dimension was $33 \mathrm{~nm}$ and the field of view (FOV) of the EUV image was $\sim 34 \times 34 \mu^{2}$. From the KE measurement, shown in Fig. $6 c$ ), it can be inferred that the 10-90\% intensity change takes place over a distance of $\sim 3$ pixels, which corresponds to $95 \mathrm{~nm}$. The half pitch spatial resolution, often quoted in such optical systems, was therefore $48 \mathrm{~nm}$.

The theoretical half-pitch spatial resolution of the microscope is given by $r_{h-p}=k \cdot \lambda / 2 \cdot N A_{F Z P}$ $=k \cdot \Delta r$, where $k$ is illumination dependent constant, NAFZP is the numerical aperture of the objective FZP $\left(N A_{F Z P}=0.137\right)$ and $\Delta r$ is the outer zone width $(\Delta r=50 \mathrm{~nm})$. For incoherent illumination $k=0.61$ and the half-pitch spatial resolution is $30.5 \mathrm{~nm}$. The theoretical resolution can only be achieved for radiation with bandwidth $\Delta \lambda_{\text {rad }}$ fulfilling spectralbandwidth requirement of the zone plate: $\Delta \lambda_{\text {rad }}<\Delta \lambda_{\mathrm{ZP}}=\lambda_{\text {rad }} / \mathrm{N}$ (N - number of zones). In our case, as mentioned in section 2.1, there are two dominant, closely spaced spectral lines for $\mathrm{Ar}^{\mathrm{VIII}}$ emission, at $\lambda=13.793 \mathrm{~nm}$ and at $\lambda=13.844 \mathrm{~nm}$ that, separately, fulfil the monocromaticity criterion. However, even though one line at $13.84 \mathrm{~nm}$ is stronger than the other one (Fig. 4), still, the other line produces its own image, slightly out of focus, which degrades the overall spatial resolution of the EUV image to $\sim 50 \mathrm{~nm}$. It could be improved by using additional filter to remove one of those lines. However, those lines are only $0.51 \AA$ apart, and the development of such filter so far is a significant challenge.

In order to investigate a non-trivial object, the sample made of silver nanowires deposited on top of $30 \mathrm{~nm}$ thick silicon nitride membrane was imaged in the EUV spectral range. The silver nanowires, with a diameter in the range of 10-100 $\mathrm{nm}$ and a length of a few $\mu \mathrm{m}$, were synthesized using $\mathrm{CuCl}_{2}$-mediated polyol process. Fig. 7 shows a detailed image of the silver nanowires obtained for comparison with a visible light (Fig. 7a) and the EUV microscope (Fig. 7b). To acquire this image 200 EUV pulses were accumulated, at a $10 \mathrm{~Hz}$ source repetition rate, and with an exposure time of 22 seconds. In the EUV image nanowires with variable lengths and thicknesses of the order of a fraction of a micron can be observed. The EUV image exhibits superior spatial resolution and much higher optical magnification (410x) compared with visible light micrograph, obtained with a NA $=0.7,40 x$ objective.

Finally, a biological sample of dehydrated silica-scaled chrysophyte alga Chrysodidymus synuroideus (Wujek, 2013; http://megasun.bch.umontreal.ca/ protists/cdid/appearance.html;

http://cfb.unh.edu/phycokey/Choices/Synurophyceae/CHRYSODIDYMUS/Chrysodidymus Image page.html) was investigated with the EUV microscope. These cells, $\sim 5 \mu \mathrm{m}$ in size, have an armour of siliceous scales, consisting of a perforated oval plate with a short apical spine. Cultured colonies consist of two cells attached. The cells were cultured in DY-V Medium (Bigelow), at an optimum growth temperature of $22{ }^{\circ} \mathrm{C}$. A drop of the algal suspension $(10 \mu \mathrm{l})$ was placed onto a $30 \mathrm{~nm}$ thick $\mathrm{Si}_{3} \mathrm{~N}_{4}$ membrane. The sample was fixed with $4 \%$ paraformaldehyde in PBS for 30 minutes at room temperature. Then the sample was rinsed with PBS 1x 5 min. Dehydration of the cells was done by series of ethanol-distilled water washes $(25 \% \mathrm{EtOH} 1 \times 5 \mathrm{~min} ; 50 \% \mathrm{EtOH} 1 \times 5 \mathrm{~min} ; 70 \% \mathrm{EtOH} 1 \mathrm{x} 5 \mathrm{~min} ; 80 \% \mathrm{EtOH}$ 1x 5 min; 95\% EtOH 1x 5 min; 100\% EtOH 1x 10 min) followed by air dry.

External structures of the cells are visible due to the phase contrast in visible light microscopy images (Fig. 7c) and as a modulation in transmittance of the EUV light through the sample in the EUV images (Fig. 7d). The EUV images were acquired with 100 EUV pulses - $12 \mathrm{sec}$. exposure, a detector temperature of $-20{ }^{\circ} \mathrm{C}$ and a CCD readout speed of 50 kHz. The EUV images, Fig. 7d, e), show enhanced spatial resolution compared with the visible light micrograph, owing to much shorter wavelengths, beyond the capabilities of diffraction limited visible light microscopes. Small features of the order of $50 \mathrm{~nm}$ in size, approaching the half-pitch spatial resolution of the EUV microscope, can be observed in Fig. $7 \mathrm{e})$. 
Although, this spatial resolution cannot compete with the SEM, it has to be noted that the EUV microscope still operates using photons (although with higher energy) as information carriers, instead of electrons. It is important, because electrons interact with matter differently than the photons, thus providing the possibility to obtain different kind of information from the samples. Moreover, although its resolution is similar to STED in the visible light region (Hanne et al., 2015), it enables the acquisition of full field images, contrary to STED acquisition, which operates in scanning mode, and does not require any fluorescence markers or staining, which modify the sample to be imaged. That is why EUV microscopy may be regarded as a complementary technique to the existing, well established imaging tools and methods, for acquisition of information about the samples, which cannot be accessed using other techniques.

\section{CONCLUSIONS}

A novel, compact, stand-alone EUV transmission microscope, based on Fresnel optic and Ar-based double stream gas-puff target EUV source, was developed and characterized. This microscope, under incoherent illumination, can image samples at a $13.8 \mathrm{~nm}$ wavelength with a half-pitch spatial resolution reaching sub-50 $\mathrm{nm}$. In addition, we have imaged a nanostructure and resolved features well below the resolution limit of conventional visiblelight bright field microscopes, thus providing a superior spatial resolution compared to visible light microscopes, without the need to employ very complicated and time consuming serial acquisition optical schemes, such as STED.

In the effort to print smaller and more energy-efficient micro- and nano-structures researchers have employed increasingly shorter wavelengths from the EUV range. The emission at $13.8 \mathrm{~nm}$ from argon was proved to be quasi-monochromatic and sufficient for the FZP to achieve sub-50 nm spatial resolution. This $13.8 \mathrm{~nm}$ wavelength radiation is close to the lithographic wavelength so it can be employed in research fields related to lithography for semiconductor applications as well as for nano-lithographic mask inspections in order to observe small features with high resolution, as well as to nanotechnology and material science. Additionally, the employment of the EUV radiation enables the study of small cellular features (such as dendrites or flagella, as shown in Fig.7e) or small biological organisms, such as diatoms, due to the high EUV contrast and absorption of EUV light in carbon.

The EUV microscopy requires no specific sample preparation, such as conductive coating for SEM and is still photon-based, which is important in certain types of applications requiring photon illumination such as electron beam photoresists, for example. The short attenuation length of EUV photons in almost any material can be used to enhance optical contrast and, as a result of the intermediate photon energy, it still produces less radiation damage than the hard X-ray beams. Thus, the EUV microscopy can be employed as a complementary imaging technique to the well-established ones, for example in thin layer studies, where it could provide additional information to optical and SEM microscopy. Such high resolution imaging systems, operating in the EUV and shorter soft X-ray spectral ranges, have already found many different applications in different areas of science and technology, such as material science, biomedicine, nanotechnology, microelectronics, among others. These compact systems, such as the one presented in this paper are of major importance because of their widespread applicability, which could promote rapid future developments in science and technology. 


\section{Acknowledgments}

This work is supported by the National Centre for Research and Development's, LIDER programme, grant \#LIDER/004/410/L-4/12/NCBR/2013 and the National Science Centre, Opus programme, grant agreement number UMO-2015/17/B/ST7/03718. The authors acknowledge also financial support from the EU FP7 Erasmus Mundus Joint Doctorate Program EXTATIC, under a framework partnership agreement FPA-2012-0033 and from the European Union's Horizon 2020 research and innovation program, under the LaserlabEurope project, grant agreement No. 654148.

\section{References}

Baez, A. V. (1952). Resolving Power in Diffraction Microscopy with Special Reference to XRays. Nature 4310, 963-964.

Baez, A. (1952). A study in diffraction microscopy with special reference to X-rays. J. Opt. Soc. Am. 42(10), 756-762.

Baez, A. V. (1961). Fresnel Zone Plate for Optical Image Formation Using Extreme Ultraviolet and Soft X Radiation. J. Opt. Soc. Am. 51(4), 405-412.

Bartnik, A., Fiedorowicz, H., Fok, T., Jarocki, R., Kostecki, J., Szczurek, A. \& Węgrzyński, Ł. (2014). Laser-plasma SXR/EUV sources: adjustment of radiation parameters for specific applications. Proceedings of SPIE 9441, 94410R.

Benk, M. P., Goldberg, K. a., Wojdyla, A., Anderson, C. N., Salmassi, F., Naulleau, P. P., \& Kocsis, M. (2015). Demonstration of 22-nm half pitch resolution on the SHARP EUV microscope. J. Vac. Sci. Technol. B, 33(6), 06FE01.

Döring, F., Robisch, L., Eberl, C., Osterhoff, M., Ruhlandt, A., Liese, T., Schlenkrich, F., Hoffmann, S., Bartels, M., Salditt, T. \& Krebs, H. U. (2013). Sub-5 nm hard x-ray point focusing by a combined Kirkpatrick-Baez mirror and multilayer zone plate. Opt. Express, 21(16), 19311-19323.

Fiedorowicz, H., Bartnik, A., Daido, H., Choi, W., Suzuki, M. \& Yamagami, S. (2000). Strong extreme ultraviolet emission from a double-stream xenon/helium gas puff target irradiated with a Nd:YAG laser, Opt. Commun., 184 (1-4), 161-167.

Fiedorowicz, H., Bartnik, A., Jarocki, R., Kostecki, J., Krzywinski , J., Mikołajczyk, J., Rakowski, R., Szczurek, A. \& Szczurek, M. (2015). Compact laser plasma EUV source based on a gas puff target for metrology applications. J. Alloy Compnd., 401(1-2), 99103.

Hädrich, S., Klenke, A., \& Rothhardt, J. (2014). High photon flux table-top coherent extreme ultraviolet source. Nat. Photonics, 8(10), 780.

Hädrich, S., Rothhardt, J., Krebs, M., Demmler, S., Limpert, J., \& Tunnermann, A. (2015). High photon flux and repetition rate table-top EUV sources based on ultrashort pulse fiber lasers. Photonics Conference (IPC),1, 72-73, IEEE.

Hanne, J., Falk, H.J., Görlitz, F., Hoyer, P., Engelhardt, J., Sahl, S.J. \& Hell, S.W. (2015). STED nanoscopy with fluorescent quantum dots. Nat. Commun., 6, 7127.

Heck, J. M., Attwood, D. T., Berkeley, E. O. L., Meyer-ilse, W., Anderson, E. H., \& Berkeley, E. O. L. (1998). Resolution determination in X-ray microscopy: an analysis of the effects of partial coherence and illumination spectrum. J. X-Ray Sci. Technol., 8, 95-104.

Jones, M. W. M., Dearnley, M. K., van Riessen, G. A., Abbey, B., Putkunz, C. T., Junker, M. D., Vine, D.J., McNulty, I., Nugent, K.A., Peele, A.G. \& Tilley, L. (2014). Rapid, low dose X-ray diffractive imaging of the malaria parasite Plasmodium falciparum. Ultramicroscopy, 143, 88-92. 
Kelly, R. L. (1987). Atomic and ionic spectrum lines below 2000 Angstroms: Hydrogen through Krypton Part I (H-Cr). J. Phys. Chem. Ref. Data. American Chemical Society and American Institute of Physics for the National Bureau of Standards.

Kirz, J. (1974). Phase zone plates for X-rays and the extreme UV. J. Opt. Soc. Am., 64(3), 301-309.

Klosner, M., \& Silfvast, W. T. (1998). Intense xenon capillary discharge extreme-ultraviolet source in the 10-16-nm-wavelength region.Opt. Lett., 23(20), 1609-11.

Korte, K. E., Skrabalak, S. E., Xia, Y. (2008). Rapid synthesis of silver nanowires through a CuCl- or $\mathrm{CuCl}_{2}$-mediated polyol process. J. Mater. Chem. 18, 437-441.

Legall, H., Stiel, H., Blobel, G., Seim, C., Baumann, J., Yulin, S., Esser, D., Hoefer, M., Wiesemann, U., Wirtz, M. (2013). A compact Laboratory Transmission X-ray Microscope for the water window. J. Phys.: Conf. Ser., 463(1), 012013.

Mey, T., Rein, M., Großmann, P., \& Mann, K. (2012). Brilliance improvement of laserproduced soft x-ray plasma by a barrel shock. New J. Phys., 14, 073045.

Mizutani, R., Taguchi, K., Ohtsuka, M., Kimura, M., Takeuchi, A., Uesugi, K., \& Suzuki, Y. (2013). X-ray microtomographic visualization of Escherichia coli by metalloprotein overexpression. J. Syncrotron Radiat., 20(4), 581-586.

Müller, M., Mey, T., Niemeyer, J., \& Mann, K. (2014). Table-top soft x-ray microscope using laser-induced plasma from a pulsed gas jet. Opt. Express, 22(19), 23489-95.

Ojeda, J., Arrell, C. A., Grilj, J., Frassetto, F., Mewes, L., Zhang, H., Van Mourik, F., Poletto, L. \& Chergui, M. (2016). Harmonium: A pulse preserving source of monochromatic extreme ultraviolet (30-110 eV) radiation for ultrafast photoelectron spectroscopy of liquids. Struct. Dyn., 3(2), 023602.

Park, I. Y., Choi, J., Lee, D. H., Han, S., Kim, S., \& Kim, S. W. (2013). Generation of EUV radiation by plasmonic field enhancement using nano-structured bowties and funnelwaveguides. Ann. Phys., 525(1-2), 87-96.

Rehbein, S., Heim, S., Guttmann, P., Werner, S., \& Schneider, G. (2009). UltrahighResolution Soft-X-Ray Microscopy with Zone Plates in High Orders of Diffraction. Phys. Rev. Lett., 103(11), 1-4.

Saiga, R., Mizutani, R., Inomoto, C., Takekoshi, S., Nakamura, N., Tsuboi, A., Osawa, M., Arai, M., Oshima, K., Itokawa, M., Uesugi, K., Takeuchi, A., Terada, Y. \& Suzuki, Y. (2016). Three-dimensional structure of brain tissue at submicrometer resolution. AIP Conf. Proc. 020004.

Simons, H., King, A., Ludwig, W., Detlefs, C., Pantleon, W., Schmidt, S., Stöhr, F., Snigireva, I., Snigirev A. \& Poulsen, H. F. (2015). Dark-field X-ray microscopy for multiscale structural characterization. Nat. Commun., 6, 6098.

Tadesse, G.K., Klas, R., Demmler, S., HÄdrich, S., Wahyutama, I., Steinert, M., Spielmann, C., ZÜrch, M., TÜnnermann, A., Limpert, J. \& Rothhardt, J. (2016). High Speed and High Resolution Table-Top Nanoscale Imaging. arXiv:1605.02909 [physics.optics].

Thibault, P., Dierolf, M., Andreas, M., Bunk, O., David, C., \& Pfeiffer, F. (2008). HighResolution Scanning X-ray Diffraction Microscopy. Science, 321(5887), 379-383.

Vaschenko, G., Brizuela, F., Brewer, C., Grisham, M., Mancini, H., Menoni, C. S., Marconi, M. C., Rocca, J. J., Chao, W., Liddle, J. A., Anderson, E. H., Attwood, D. T., Vinogradov, A. V., Artioukov, I. A., Pershyn, Y. P. \& Kondratenko, V. V. (2005). Nanoimaging with a compact extreme-ultraviolet laser. Opt. Lett., 30(16), 2095-7.

Vaschenko, G., Brewer, C., Brizuela, F., Wang, Y., Larotonda, M. A., Luther, B. M., Marconi, M. C., Rocca, J. J., Menoni, C. S., Anderson, E. H., Chao, W., Harteneck, B. D., Liddle, J. A., Liu, Y. \& Attwood, D. T. (2006). Sub-38nm resolution tabletop microscopy with 13nm wavelength laser light. Opt. Lett., 31(9), 1214. 
Wachulak, P. W., Capeluto, M.G., Marconi, M. C., Patel, D., Menoni, C. S. \& Rocca, J.J (2007). Nanoscale patterning in high resolution HSQ photoresist by interferometric lithography with table top EUV lasers. J. Vac. Sci. Technol. B, 25(6), 2094.

Wachulak, P. W., Brewer, C. A., Brizuela, F., Menoni, C. S., Chao, W., Anderson, E. H., Bartels, R. A., Rocca, J. J. \& Marconi, M. C. (2008). Analysis of extreme ultraviolet microscopy images of patterned nanostructures based on a correlation method. J. Opt. Soc. Am. B, 25(7), B20.

Wachulak, P. W., Marconi, M. C., Bartels, R. A., Menoni, C. S. \& Rocca, J. J. (2009). Sub50nm Extreme ultraviolet holographic imaging. Proceedings of SPIE 7358, 735806735806-8.

Wachulak, P. W., Bartnik, A. \& Fiedorowicz, H. (2010). Sub-70 nm resolution tabletop microscopy at $13.8 \mathrm{~nm}$ using a compact laser-plasma EUV source. Opt. Lett., 35(14), 2337-2339.

Wachulak, P. W., Bartnik, A., Fiedorowicz, H., Paenek, D. \& Bruza, P. (2012). Imaging of nanostructures with sub-100 nm spatial resolution using a desktop EUV microscope. App. Phys. B, 109(1), 105-111.

Wachulak, P. W., Bartnik, A., Baranowska-Korczyc, A., Panek, D., Bruza, P., Kostecki, J., Węgrzyński, Ł., Jarocki, R., Szczurek, M., Fronc, K., Elbaum, D. \& Fiedorowicz, H. (2013). Study of crystalline thin films and nanofibers by means of the laser-plasma EUV-source based microscopy. Rad. Phys. Chem., 93, 54-58.

Wachulak, P. W., Wegrzynski, L., Záprazný, Z., Bartnik, A., Fok, T., Jarocki, R., Kostecki, J., Szczurek, M., Korytár, D \& Fiedorowicz, H. (2014). Extreme ultraviolet tomography using a compact laser-plasma source for 3D reconstruction of low density objects. Opt. Lett., 39(3), 532-535.

Wachulak, P. W., Torrisi, A., Bartnik, A., Węgrzyński, Ł., Fok, T., Jarocki, R., Kostecki, J., Szczurek, M. \& Fiedorowicz, H. (2015). Fresnel zone plate telescope for condenser alignment in water-window microscope. J. Opt., 17(5), 055606.

Wachulak, P. W., Torrisi, A., Bartnik, A., Adjei, D., Kostecki, J., Wegrzynski, L., Jarocki, R., Szczurek, M. \& Fiedorowicz, H. (2015). Desktop water window microscope using a double-stream gas puff target source. App. Phys. B, 118(4), 573-578.

Wachulak, P. W., Bartnik, A., Kostecki, J., Wegrzynski, L., Fok, T., Jarocki, R., Szczurek, M. \& Fiedorowicz, H. (2015). Extreme ultraviolet and soft X-ray imaging with compact, table top laser plasma EUV and SXR sources. Nucl. Instr. Meth. B, 364, 40-48.

Wilke, R. N., Priebe, M., Bartels, M., Giewekemeyer, K., Diaz, A., Karvinen, P. \& Salditt, T. (2012). Hard X-ray imaging of bacterial cells: nano-diffraction and ptychographic reconstruction.Opt. Express, 20(17). 19232.

Wujek, D. E. (2013). Silica-Scaled Chrysophytes (Chrysophyceae and Synurophyceae) from New Zealand Freshwaters II Additions to the Flora. Pac. Sci., 67(1), 113-118.

Yase, S., Nagaya, K., Mizoguchi, Y., Yao, M., Fukuzawa, H., Motomura, K., Yamada, A., Ma, R., Ueda, K.,Saito, N., Nagasono, M., Togashi, T., Tono, K., Yabashi, M., Ishikawa, T., Ohashi, H. \&Senba, Y. (2013). Crossover in the photoionization processes of neon clusters with increasing EUV free-electron-laser intensity. Phys. Rev. A, 88(4), $1-5$.

Zhang, B., Seaberg, M. D., Adams, D. E., Gardner, D. F., Shanblatt, E. R., Shaw, J. M., Chao, W., Gullikson, E.M., Salmassi, F., Kapteyn, H.C. \& Murnane, M. M. (2013). Full field tabletop EUV coherent diffractive imaging in a transmission geometry. Opt. Express, 21(19), 839-843. 
a)
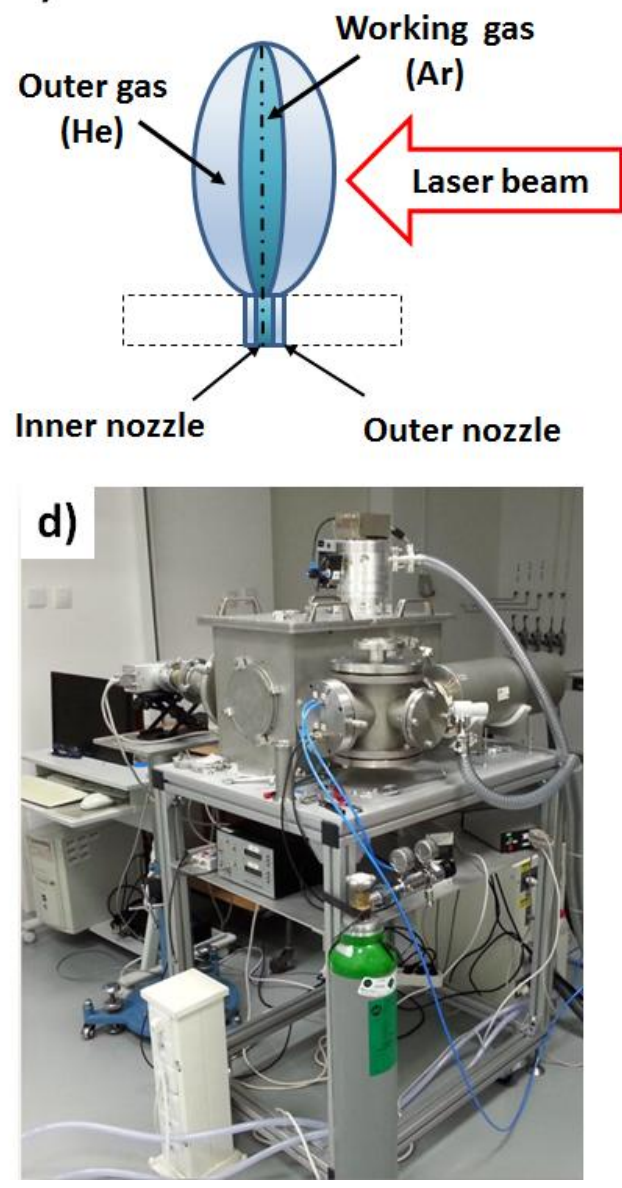

b)
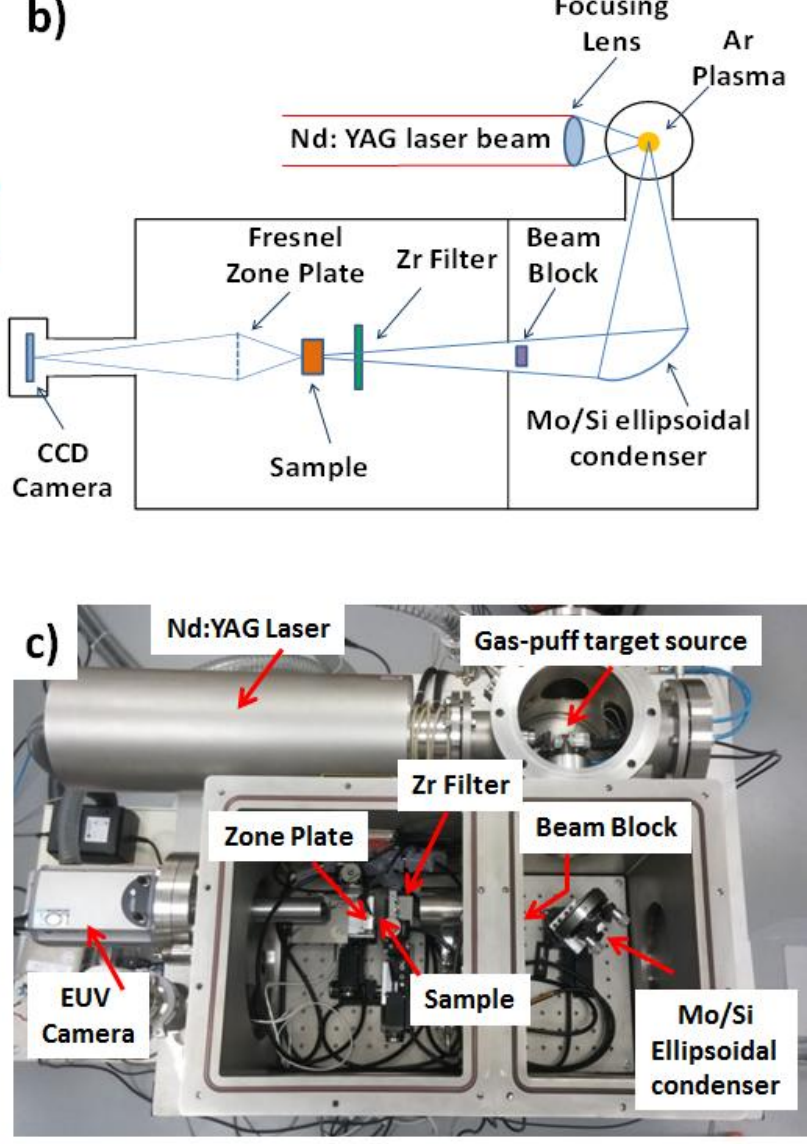

Fig. 1. Scheme of the double stream gas-puff target (a), scheme of the microscope (b), photo showing internal components of the EUV microscope (c) and photo of the entire microscopy system (d). 


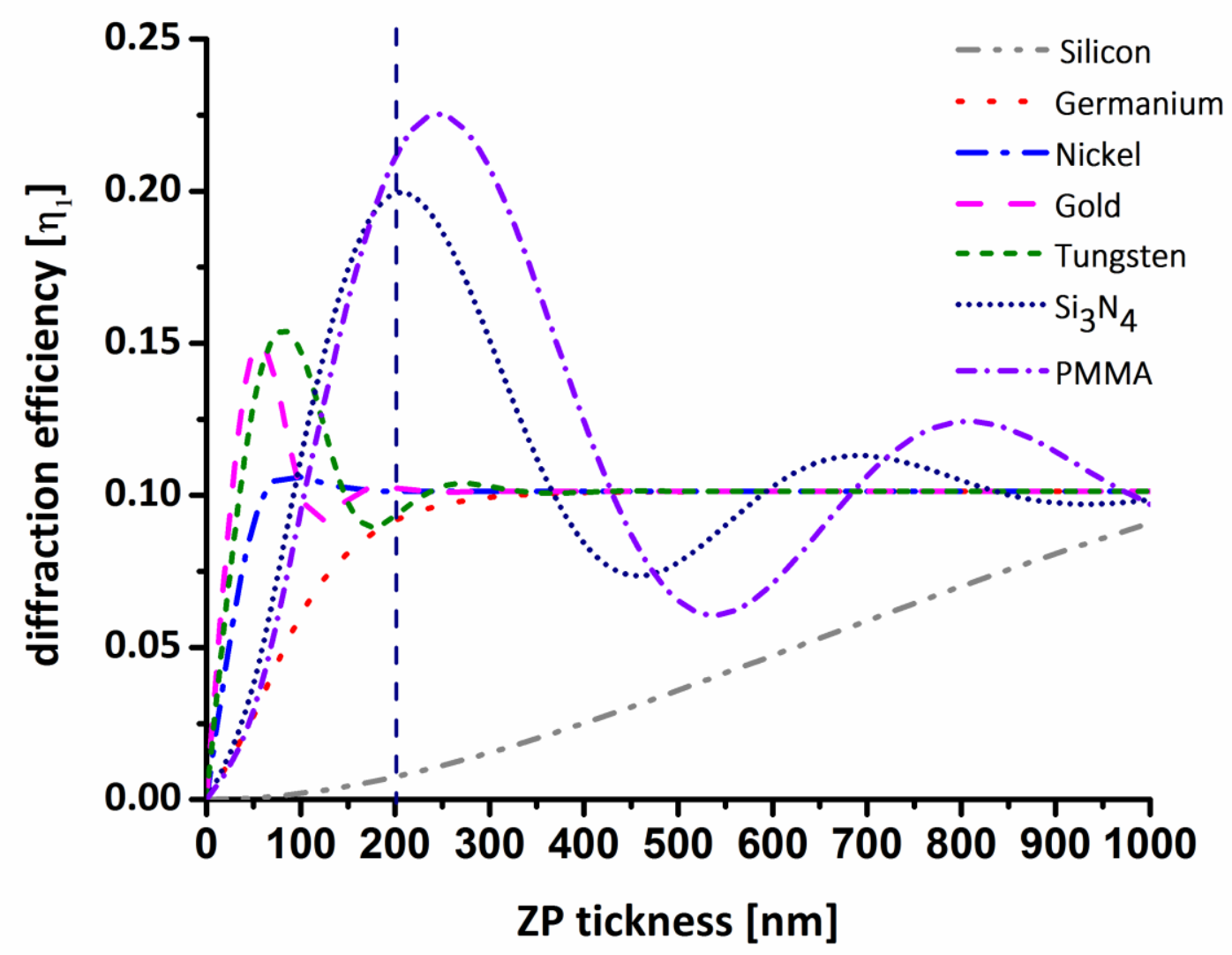

Fig. 2. Fresnel zone plate diffraction efficiency in the first diffraction order for different materials commonly used for FZP fabrication as a function of the thickness of the lens at $\lambda=13.84 \mathrm{~nm}$. 

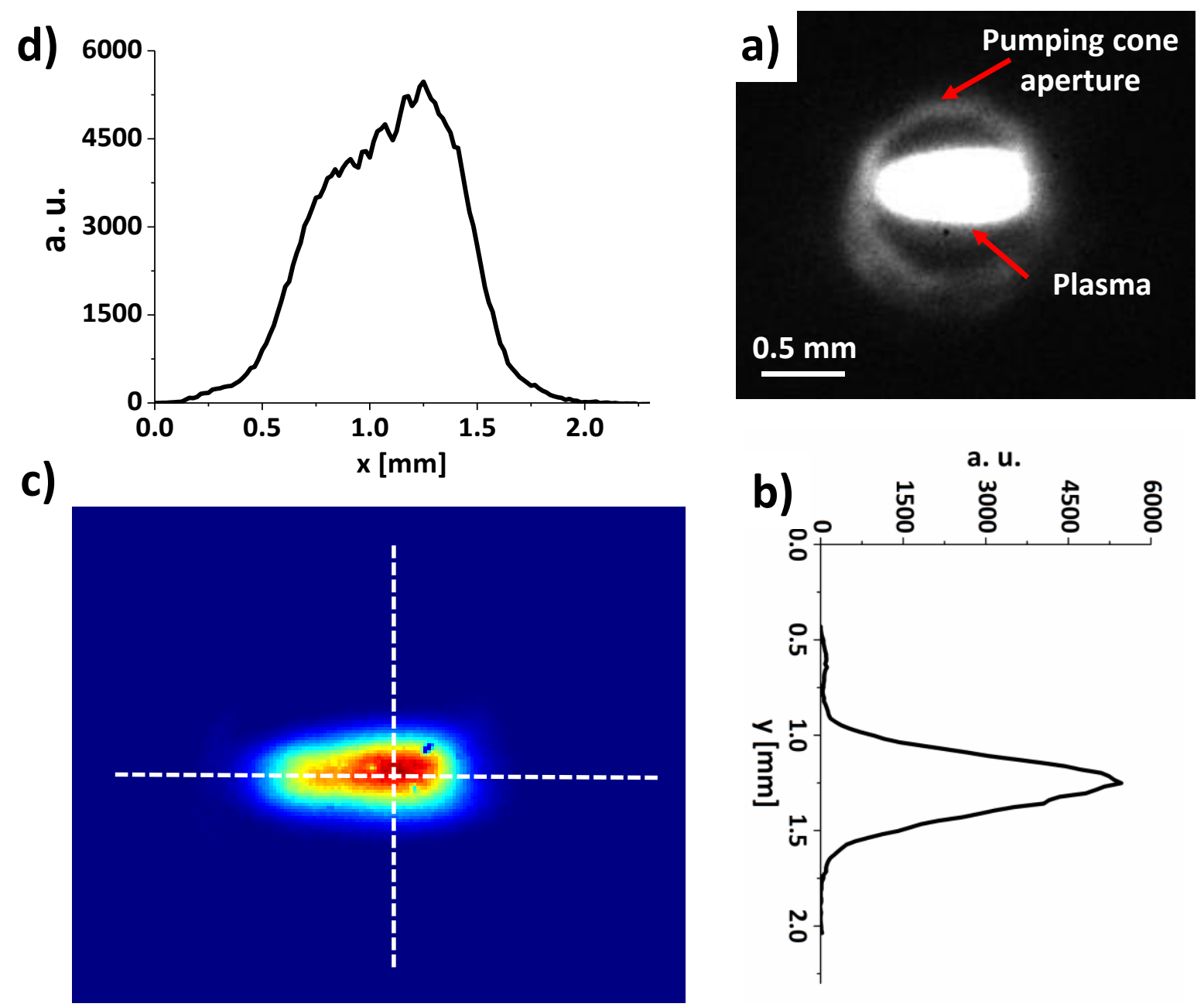

Fig. 3. Ar plasma image obtained with a pinhole camera (a). The brightness was deliberately raised to show the plasma surrounded by a thin symmetrical ring with a diameter of $1.2 \mathrm{~mm}$, created by the scattering of plasma radiation from the pinhole edge in the differential pumping cone. The plasma profile in two orthogonal directions, acquired after $20 \mathrm{EUV}$ shots using a $250 \mathrm{Zr}$ filter is shown in (b,d). (c), in "false colors", shows in detail the most intense regions of plasma that emits EUV radiation (red). The plasma is elliptic in shape and is elongated in the direction of incidence of the $\mathrm{Nd}$ :YAG laser beam. 


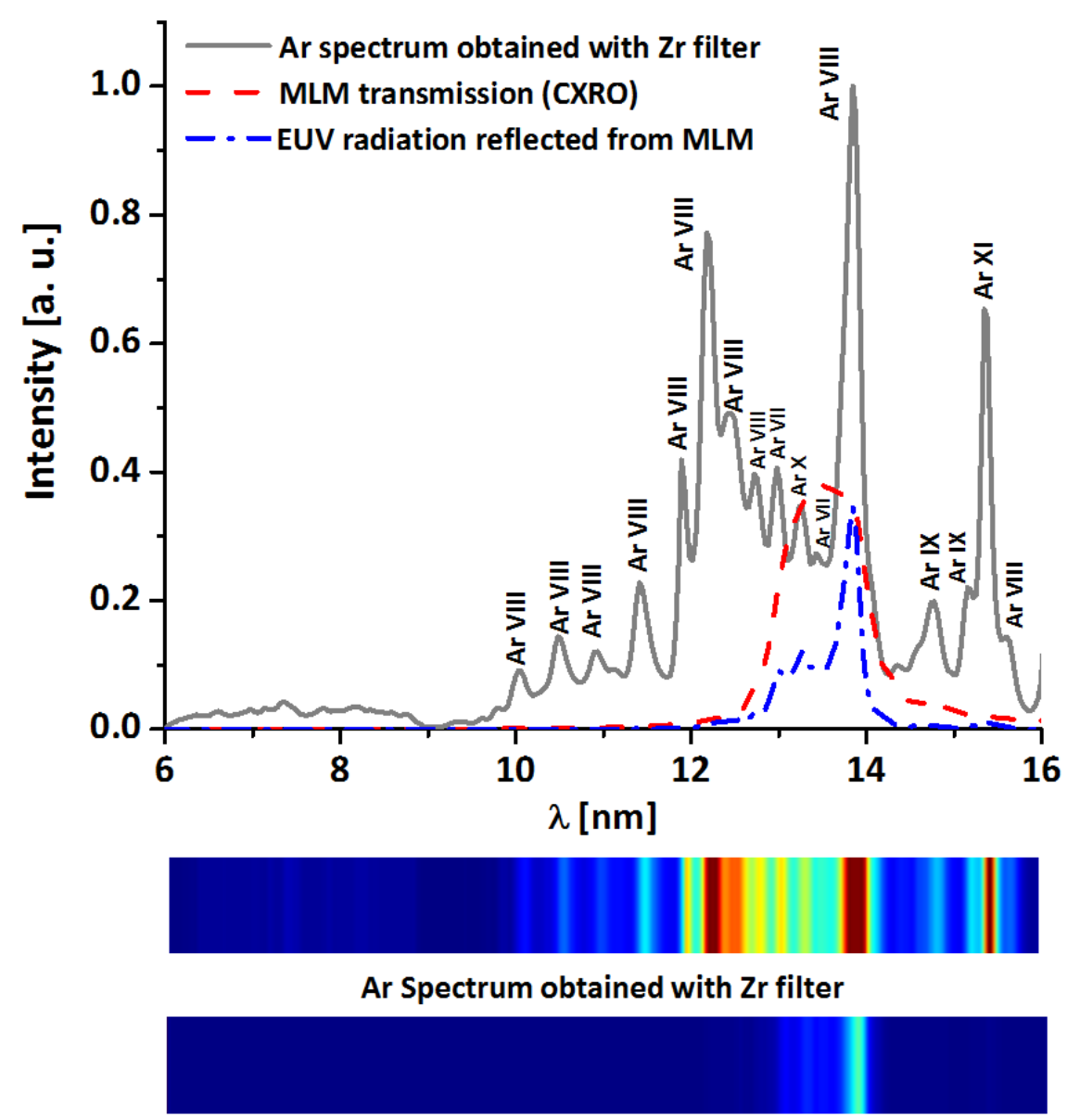

EUV radiation reflected from MLM

Fig. 4. Spectral measurement of the Ar plasma emission (grey line) acquired with 20 EUV pulses and employing a $250 \mathrm{~nm}$ thick $\mathrm{Zr}$ filter to remove longer wavelengths. Radiation from the plasma was focused and filtered using the multilayer condenser mirror (MLM), which transmission is shown in dashed-red line. The dash-dotted blue line shows the total contribution in the wavelength range 13-14 $\mathrm{nm}$ (reflected from the MLM condenser), which is used for subsequent imaging. 


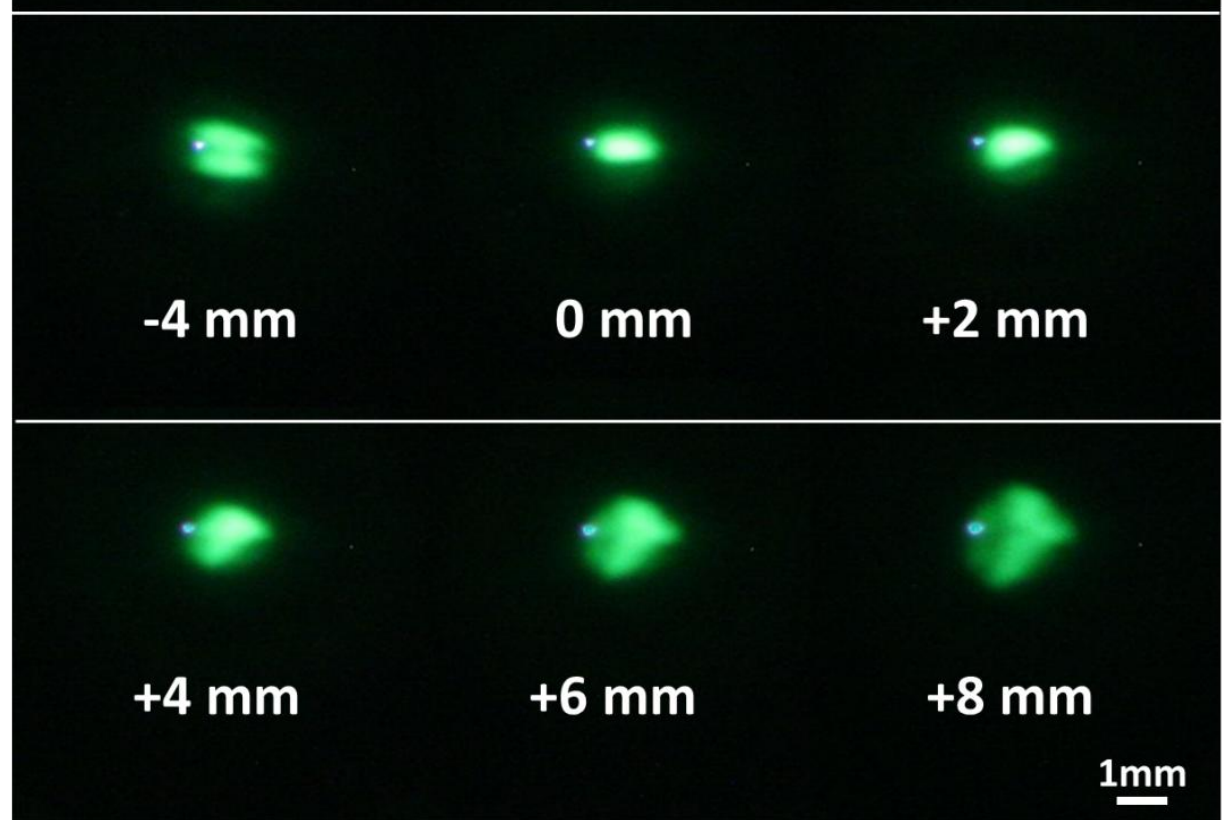

Fig. 5. Images of the spatial distribution of radiation focused by the condenser in the proximity of the condenser second focal plane. The distances show displacement of the measurement plane from the optimal in-focus position at $\mathrm{z}=0 \mathrm{~mm}$. The smallest and most uniform achievable spot (central image) was ellipsoidal, elongated in the horizontal direction, $1.5 \mathrm{x} 0.8 \mathrm{~mm}^{2}$ (FWHM) in size. 

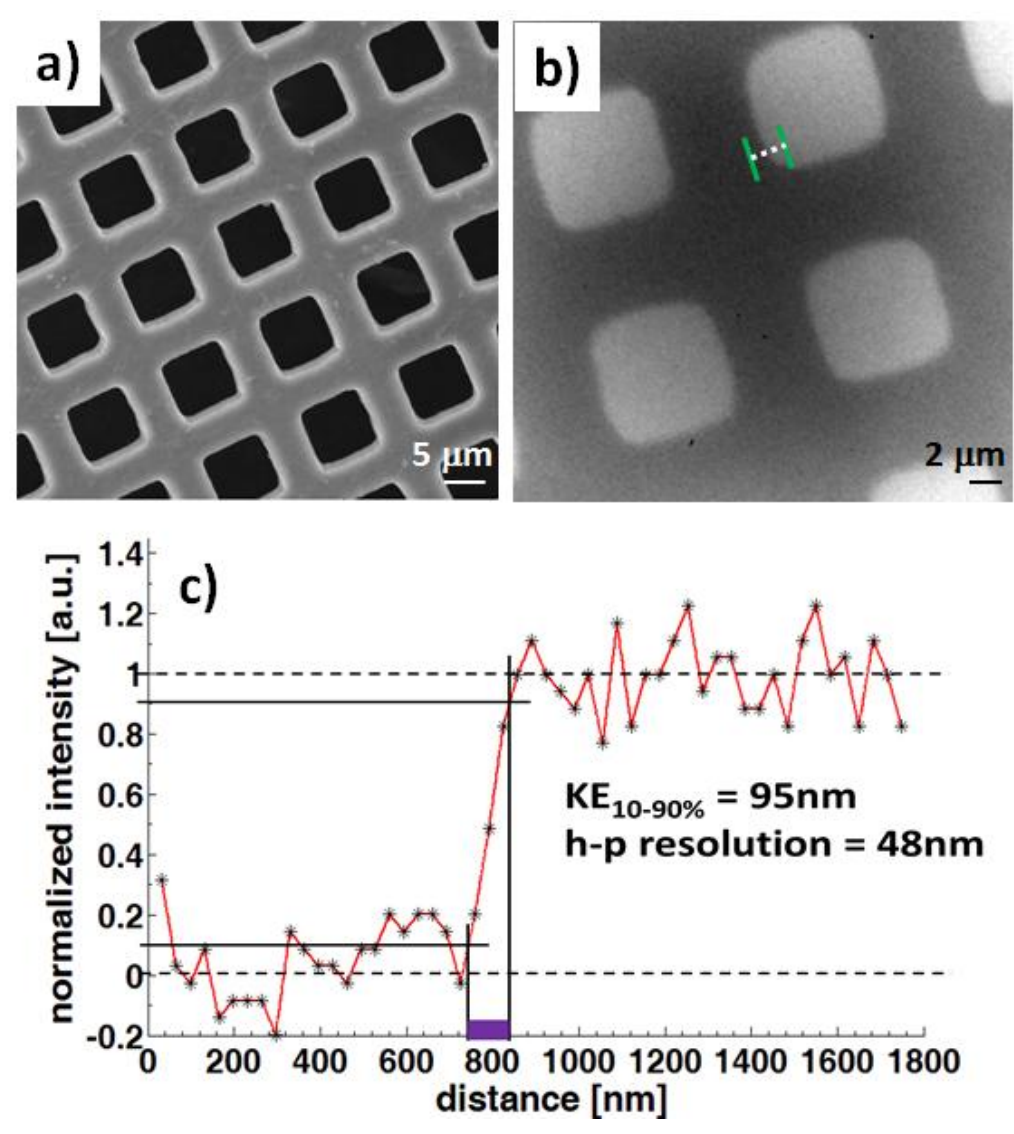

Fig. 6. Comparison of mesh image acquired by the SEM (a) and the EUV microscope (b). Knife-edge spatial resolution test for the EUV image (c). The estimated 10\% to $90 \%$ intensity transition across the sharp edge is equal to $95 \mathrm{~nm}$ suggesting half-pitch spatial resolution of $48 \mathrm{~nm}$. The KE profile was obtained in region marked with a dotted white line between green markers. 

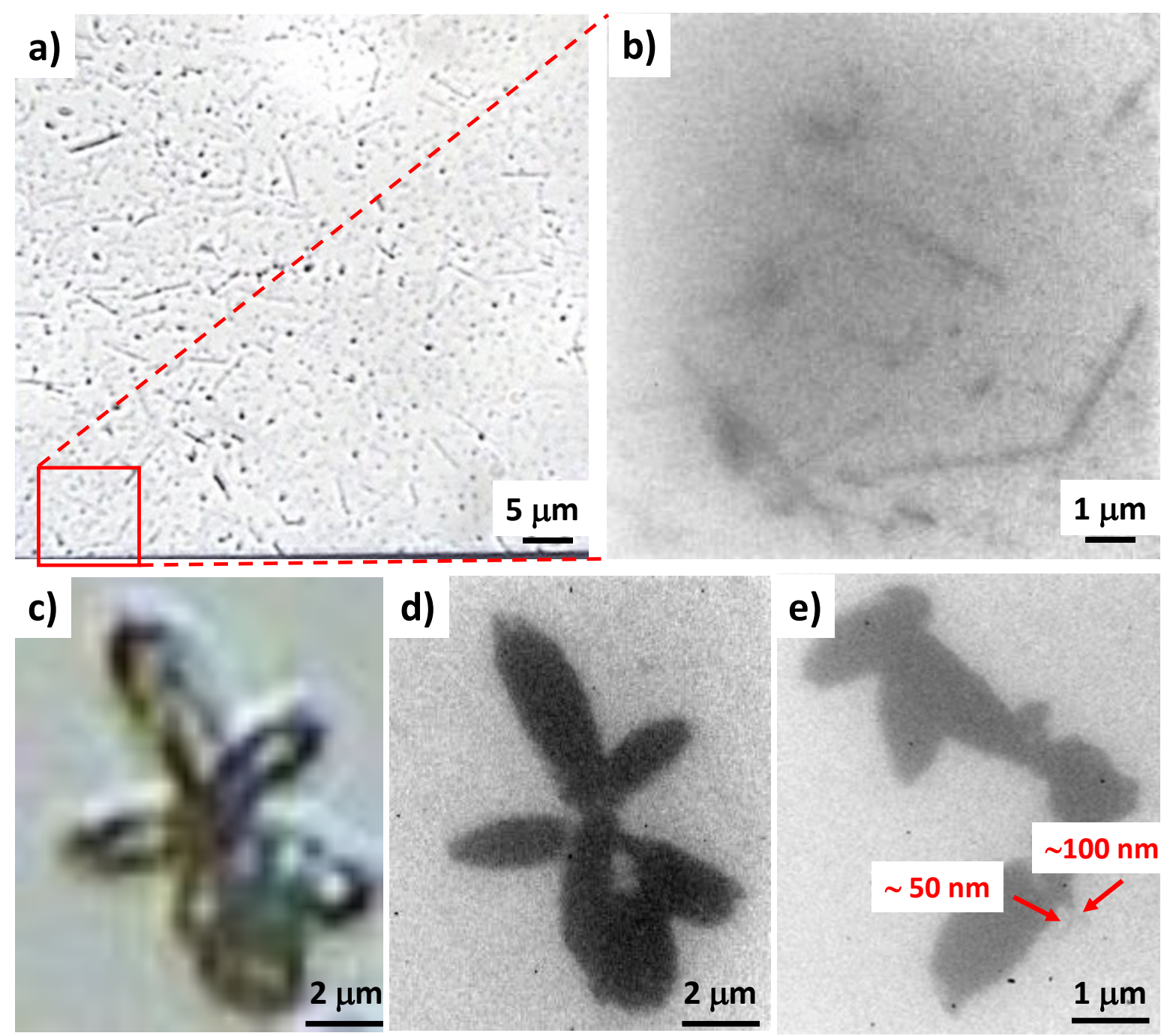

Fig. 7. Comparison of images of silver nanowires deposited on top of $30 \mathrm{~nm}$ thick silicon nitride membrane, imaged with visible light (40x objective, $N A=0.7)$ (a) and the EUV microscope (410x objective magnification, NA=0.137) (b). The high resolution of the EUV microscope enhances details and features smaller than a fraction of a micron. Figs (c) and (d) show a direct comparison of Chrisodidymus cells, imaged with the visible and the EUV microscope. The EUV microscope permits to observe, in the same type of sample, very small features, approaching the resolution limit of the microscope (e). 